\title{
Superfluid swimmers
}

\author{
G. V. Kolmakov ${ }^{1}$ and I. S. Aranson $\oplus^{2}$ \\ ${ }^{1}$ Physics Department, New York City College of Technology, The City University of New York, Brooklyn, New York 11201, USA \\ ${ }^{2}$ Departments of Biomedical Engineering, Chemistry, and Mathematics, Pennsylvania State University, \\ University Park, Pennsylvania 16802, USA
}

(Received 9 November 2020; accepted 10 February 2021; published 26 February 2021)

\begin{abstract}
The propulsion of living microorganisms ultimately relies on viscous drag for body-fluid interactions. The selflocomotion in superfluids such as ${ }^{4} \mathrm{He}$ is deemed impossible due to the apparent lack of viscous resistance. Here, we investigate the self-propulsion of a Janus (two-face) light-absorbing particle suspended in superfluid helium ${ }^{4} \mathrm{He}(\mathrm{He}-\mathrm{II})$. The particle is energized by the heat flux due to the absorption of light from an external source. We show that a quantum mechanical propulsion force originates due to the transformation of the superfluid to a normal fluid on the heated particle face. The theoretical analysis is supported by the numerical solution of the Ginzburg-Landau-Khalatnikov model for a superfluid. Our results shed light on the dynamics of inclusions in a superfluid and stimulate experiments.
\end{abstract}

DOI: 10.1103/PhysRevResearch.3.013188

\section{INTRODUCTION}

Living organisms metabolize the chemical energy of the nutrient into self-propelled mechanical motion. Swimming in a viscous fluid, especially at low Reynolds numbers, requires complex body deformations and nonreciprocal motion [1]. Even for large animals, viscous dissipation is crucial, and strictly speaking, self-locomotion is impossible in an ideal nonviscous environment. Namely, there will be no momentum exchange between the swimmer and the fluid [2-4]. While the viscosity of the fluid is unimportant for the flow in the bulk, it is crucial for producing a thin boundary layer and skin friction at the body surface [2].

Growing needs for targeted drug delivery and flow manipulation at the microscale has stimulated progress in synthetic microswimmers [5]. The last decade featured a remarkable diversity of self-propelled microswimmer designs, from bimetallic nanorods powered by the catalytic decomposition of $\mathrm{H}_{2} \mathrm{O}_{2}$ [6], to bubble [7], acoustic [8], and enzyme propulsion [9]. Propelling colloidal Janus (two-faced) particles by light is one of the common techniques in soft matter [10-12]. The motion is often caused by self-thermophoresis: Absorption of laser light at the metal-coated side of the particle creates a local temperature gradient which propels the particle $[10,13]$.

A superfluid can be often modeled as a two-fluid mixture: dissipationless superfluid dissolved in a "normal fluid" similar to a Newtonian liquid [14]. The onset of dissipation [15-18] and the lift force [19] in superfluids received significant attention. However, the possibility of self-locomotion of a microscopic colloidal particle in superfluids was not examined.

Published by the American Physical Society under the terms of the Creative Commons Attribution 4.0 International license. Further distribution of this work must maintain attribution to the author(s) and the published article's title, journal citation, and DOI.
This situation arises when studying the motion of colloidal tracers or ice crystals in superfluid turbulence [20,21]. Light illumination used for visualization may cause nonuniform heating of the tracer and generation of a superfluid backflow. The quantum mechanical transformation of the superfluid to a normal fluid will lead to the onset of friction, and consequently, to viscous drag, resulting in particle self-motion. This problem is different from swimming in an ideal fluid $[2,3]$ due to the quantum mechanism of dissipation.

Here, we study the self-propelled motion of a Janus microparticle in superfluid ${ }^{4} \mathrm{He}$ (He-II). The particle has two faces: dark, light absorbing, and white, light reflecting [Fig. 1(a)]. Laser light irradiation results in preferential heating of the dark face. The particle moves due to "quantum thermophoresis": Generated by heat, a normal fluid exerts a propulsion force on the particle. We support this mechanism by the numerical study of particle dynamics in a model of superfluid described by the Ginzburg-LandauKhalatnikov equation (GLKE) $[18,22,23]$. The computation modeling revealed that the particle might exhibit complex dynamics characterized by long acceleration periods terminated by abrupt slowdowns and dissipation events. During the slowdown events, the energy is dampened, and the particle releases quantum vortices, generating second sound. Our findings provide insights into the dynamics of macroscopic inclusions in a superfluid and stimulate experiments. We also connect the seemingly unrelated hard condensed matter topic of superfluidity and the rapidly expanding field of active matter [24,25]. Furthermore, self-propelled colloids can be used as microscopic probes of the superfluid helium state.

\section{PROPULSION MECHANISM AND THE ORIGIN OF TRACTION}

The radiation of heat to the superfluid liquid from a solid results in a force applied to the latter [23]. This force has a quantum origin and is different from the thermophoresis 

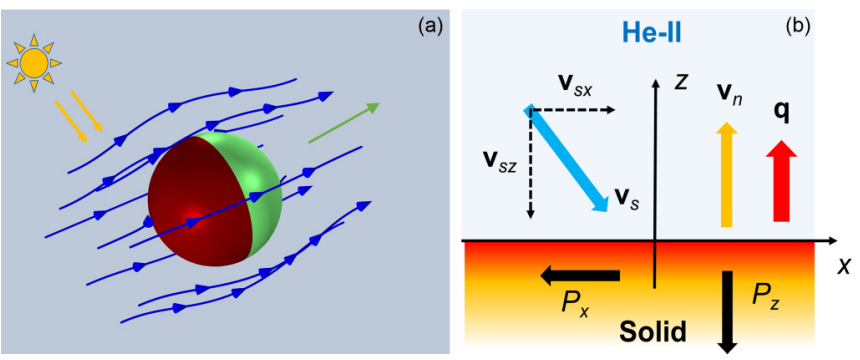

FIG. 1. (a) Schematics of a superfluid swimmer. The dark side of a Janus particle is heated by an external light source. The green arrow indicates the direction of motion, and blue arrows illustrate the superflow. (b) Flows and forces in the frame of reference moving with the solid body element. The axes $x$ and $z$ are directed along and perpendicular to the solid body surface, respectively. The heat flux $\mathbf{q}$ from the surface is directed toward the superfluid helium (He-II). The normal velocity $\mathbf{v}_{n}$ directed along $\mathbf{q}$ and the tangential component of the superfluid velocity $v_{s x}$ cause the perpendicular and tangential quantum thermophoresis forces, Eqs. (1) and (2), respectively.

force known in classical liquids or gases [26]. In "classical" thermophoresis, the particle motion is caused by a thermal gradient in the surrounding fluid. In stark contrast, for thermophoresis in a superfluid liquid, the thermal gradients are negligibly small due to extremely high convection thermal conductivity in the bulk liquid [27]. We refer to thermophoresis in a superfluid as quantum thermophoresis. The origin of the thermophoresis force can be understood in terms of the two-component GLKE of the superfluidity [28]. Namely, a heated surface irradiates quasiparticles, phonons, and rotons, which propagate from the solid to the superfluid bulk. This quasiparticle flux is the flow of the normal component of the superfluid, carrying the linear momentum away from the solid. In effect, the solid experiences a "recoil," which is the quantum thermophoresis force. In the two-component model, the force is produced by the transformation of a superfluid component (moving toward the solid surface) to a normal component (moving from the surface) at the heated body's surface [Fig. 1(b)].

The stress, i.e., the force $\mathbf{P}$ on the unit area due to quantum thermophoresis, is calculated via the momentum tensor of the superfluid $\Pi_{i k}=\rho_{s} v_{s i} v_{s k}+\rho_{n} v_{n i} v_{n k}$ as $P_{i}=\sum_{k} \Pi_{i k} n_{k}$, where $\Pi_{i k}$ is taken in the frame of reference moving with the element of the solid surface. Here, $\rho_{s(n)}$ and $\mathbf{v}_{s(n)}$ are the respective superfluid (normal) components of the density and fluid velocity, $\mathbf{n}$ is the vector perpendicular to the solid boundary element and directed toward the superfluid, and $i, k$ mark the spatial components. In the momentum tensor $\Pi_{i k}$, we omit the hydrostatic pressure since we consider it constant in the superfluid bulk. This approximation [28] follows from the smallness of the thermal expansion coefficient of superfluid helium, $(T / \rho)(\partial \rho / \partial T) \ll 1[27]$.

The thermophoresis force component perpendicular to the surface element can be found as $\Pi_{z z}$, in which the absence of the mass transfer through the surface, $j_{z}=0$, is taken into account as a boundary condition $\left(\mathbf{j}=\rho_{s} \mathbf{v}_{s}+\rho_{n} \mathbf{v}_{n}\right.$ is the mass flux of the superfluid liquid). The normal velocity $\mathbf{v}_{n}$ in the superfluid liquid is expressed via the heat (energy) flux as $\mathbf{q}=$
$S T \mathbf{v}_{n}[14]$ that gives the following,

$$
P_{z}=-\frac{\rho \rho_{n}|\mathbf{q}|^{2}}{\rho_{s} S^{2} T^{2}} .
$$

In Eq. (1), $S$ and $T$ are the respective entropy per unit volume and temperature of the fluid. In what follows we consider small energy fluxes $\mathbf{q}$ and disregard the dependence of the thermodynamic quantities on $\mathbf{q}$ as well as on the relative velocity $\mathbf{w}=\mathbf{v}_{n}-\mathbf{v}_{s}[28]$

While the normal velocity at the solid boundary is equal to the velocity of the boundary element, $\mathbf{v}_{n}=\mathbf{u}$, the boundary condition for the superfluid velocity corresponds to that for an ideal fluid [28]. A moving superfluid liquid in the presence of the flux perpendicular to the boundary exerts a tangential force on the latter. The tangential force per unit area is equal to $\Pi_{x z}=\rho_{n} v_{n z}\left(v_{n x}-v_{s x}\right)$ that gives $P_{x}=\frac{\rho_{n}|\mathbf{q}|}{S T}\left(v_{n x}-v_{s x}\right)$. We assume that the heat flux $\mathbf{q}$ is irradiated perpendicular to the solid boundary.

We also assume that the energy flux $\mathbf{q}$ is small. The extreme limit for $\mathbf{q}$ is set by the transition from the superfluid to a normal state in the liquid layer at the solid surface at large $\mathbf{q}$ [29-31]. In the small q-limit, the propelled microparticle moves with the velocities that are small compared to the first (pressure-wave) sound velocity. As follows from our simulations below, the particles can reach the critical velocities for quantized vortex generation. This velocity depends on the size of the particles and can vary from $\mathrm{cm} / \mathrm{s}$ to a few $\mathrm{m} / \mathrm{s}$ $[32,33]$. In these relatively small fluxes, accounting for the normal component velocity $v_{n}$ (which itself is of the order of $q$ ) in the tangential force $P_{x}$ results in additional damping coefficients for translational and rotational motion of the particle, proportional to $q$. We consider the damping coefficients as finite values caused by the interactions of the particle with the normal component, as explained below. Therefore, we disregard the modification of the damping coefficients proportional to small $|\mathbf{q}|$. This tangential traction force thus becomes equal to

$$
P_{\tau}=-\frac{\rho_{n}|\mathbf{q}|}{S T} v_{s x} .
$$

The tangential superfluid velocity $v_{s x}$ at the solid surface can be of the order of critical speed and is not explicitly determined by the heat flux, whereas the normal velocity vanished. Correspondingly, for small $q$ values we neglect the perpendicular traction $P_{z} \sim O\left(q^{2}\right)$ given by Eq. (1) compared to the tangential traction $P_{x} \sim O(q)$.

\section{GINZBURG-LANDAU-KHALATNIKOV EQUATIONS FOR SUPERFLUID}

We consider the two-fluid hydrodynamics of a superfluid near the $\lambda$ point, i.e., $T \approx T_{\lambda}$, formulated in terms of the GLKE for the order parameter $\psi[14,18,22,23]$,

$$
\begin{aligned}
i \hbar \partial_{t} \psi= & -\frac{\hbar^{2}}{2 m^{2}} \nabla^{2} \psi+\left(\mu_{n}+\mu_{s}\right) m \psi \\
& -\Lambda i\left[\frac{1}{2}\left(\frac{i \hbar}{m} \nabla-\mathbf{v}_{n}\right)^{2} \psi+\mu_{s}\right] m \psi,
\end{aligned}
$$


where $m$ is the mass of the helium atom, $\mu_{n}, \mu_{s}$ the chemical potential of normal and superfluids, and $\Lambda \sim \epsilon^{-1 / 3}$ is the parameter characterizing the relaxation rate that diverges near $T_{\lambda}, \epsilon=1-T / T_{\lambda}$. The superfluid velocity and density are $\mathbf{v}_{s}=\frac{\hbar}{m} \nabla \chi, \rho_{s}=m|\psi|^{2}, \chi=\arg \psi$. Near the $\lambda$ point and after appropriate scaling, Eq. (4) can be written as

$$
\partial_{t} \psi=(\Lambda+i)\left(\nabla^{2} \psi+b \psi-|\psi|^{2} \psi\right) .
$$

We neglected for simplicity the motion of the normal-fluid component since the superfluid moves much faster. However, the normal-fluid component contributes to nonzero translational and rotational drag coefficients. Near the critical temperature $T_{\lambda}$, the order parameter evolution is governed by the relaxation rate $\Lambda$. Explicit incorporation of the flow of the normal component will introduce significant computational challenges without changing the dynamics qualitatively [18].

The length is scaled by the temperature-dependent coherence length $\xi$, and the superfluid velocity by $\mathbf{v}_{s} \rightarrow$ $\mathbf{v}_{s} / v_{e}$, where $v_{e}=\hbar / m \xi$. Time is scaled by $t \rightarrow t / 2 t_{e}, t_{e}=$ $\xi / v_{e}$. Near $T_{\lambda}$, the coherence length behaves as $\xi \approx 1.63 \times$ $10^{-8} \epsilon^{-2 / 3} \mathrm{~cm}$ [34]. Detailed scaling of Eq. (4) is given in Ref. [18]. Similar equations are obtained for cold gases near the BCS-BEC transitions [35,36]. In dimensionless units, the superfluid velocity is simply $\mathbf{v}_{s}=\nabla \chi$. To incorporate the solid particle, $b$ assumes the value $b=1$ outside the particle and $b=-1$ inside the particle. As a result, the superfluid order parameter $\psi \rightarrow 0$ inside the particle.

We consider a two-dimensional (2D) model where the order parameter depends on a 2D position $\mathbf{r}$ and time $t$. The particle dynamics is governed by the Newton equations of motion for translational and rotational motion,

$$
m_{\mathrm{p}} \frac{d^{2} \mathbf{r}}{d t^{2}}+\eta \frac{d \mathbf{r}}{d t}=\mathbf{F}, \quad I_{\mathrm{p}} \frac{d^{2} \theta}{d t^{2}}+\eta_{\mathrm{R}} \frac{d \theta}{d t}=\mathbf{T},
$$

where $m_{\mathrm{p}}, I_{\mathrm{p}}$ are the mass and moment of inertia of the particle, $a$ is the particle radius, $\eta=6 \pi v a, \eta_{\mathrm{R}}=8 \pi v a^{3}$ are the translational and rotational viscous drag coefficients, $v$ is the dynamic viscosity of the normal component, and $\mathbf{F}, \mathbf{T}$ are the drag force and the torque exerted by the superfluid liquid on the particle. The force $\mathbf{F}$ and torque $\mathbf{T}$ are obtained by integrating the tangential traction $\mathbf{P}_{\tau}$ given by Eq. (2) over the surface of the particle. In the following, we divide Eqs. (5) by $m_{\mathrm{p}}, I_{\mathrm{p}}$ correspondingly. By using the same scaling for $t, \mathbf{r}$ as for the GLKE (4), we obtain from Eqs. (5) in dimensionless form,

$$
\begin{gathered}
\frac{d^{2} \mathbf{r}}{d t^{2}}+\tilde{\eta} \frac{d \mathbf{r}}{d t}=Q \int_{A} d A \mathbf{P}_{\tau}, \\
\frac{d^{2} \theta}{d t^{2}}+\frac{10}{3} \tilde{\eta} \frac{d \theta}{d t}=\frac{5 \xi}{2 a} Q \int_{A} d A \mathbf{n} \times \mathbf{P}_{\tau} .
\end{gathered}
$$

Here, $\tilde{\eta}=\frac{9}{2} \eta t_{e} / \rho_{\mathrm{p}} a^{2}$ is the dimensionless viscous drag coefficient, $\rho_{p}$ is the particle density, $A$ is the surface element, and $\mathbf{n}$ is the unit perpendicular vector to the surface. Surface integration in Eqs. (6) and (7) is performed over the dark side of the particle. In these notations, the magnitude of normalized heat flux is of the form

$$
Q=\frac{3 q}{4 \pi S T v_{e}} \frac{\rho_{n} \xi^{3}}{\rho_{\mathrm{p}} a^{3}} .
$$
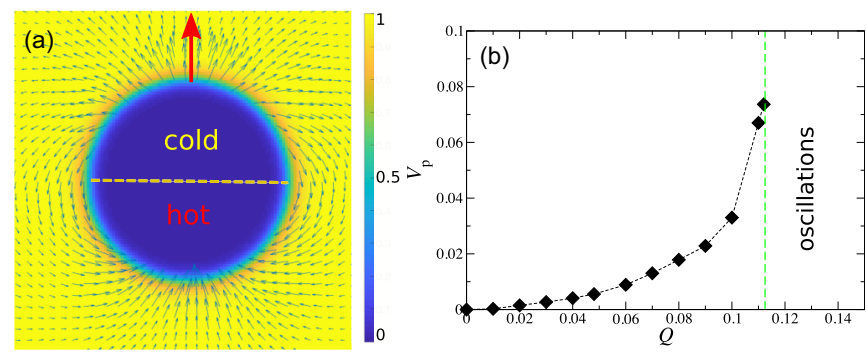

FIG. 2. (a) Superflow around the particle; the color code illustrates the distribution of $|\psi|^{2}$ (see color bar). The particle radius is $a=10 \xi$, normalized heat flux $Q=0.08, \Lambda=0.1$. The red arrow denotes the direction of motion. (b) Dependence of steady-state particle velocity $V_{\mathrm{p}}$ vs heat flux magnitude $Q$. For $Q>0.112$ the steady-state motion becomes unstable and velocity oscillations set in. Here, $a=10 \xi, \tilde{\eta}=0.2$.

The number $Q$ is the ratio of quantum thermophoretic force $\sim q$ to particle inertial force $\sim m_{\mathrm{p}}$. In these dimensionless units, the tangential traction is simply $\mathbf{P}_{\tau}=-\mathbf{v}_{s}$. Since the heat flux $\mathbf{q}$ is perpendicular to the particle surface and constant over the dark face of the particle, the surface integration of $\mathbf{P}_{\tau}$ gives a nonzero result.

\section{RESULTS}

\section{A. Dynamics of an individual particle}

Select results are shown in Figs. 2 and 3. For small values of the normalized heat flux $Q$, the steady self-propelled motion is observed with the velocity gradually increasing with $Q$ (Fig. 2). The corresponding distribution of the superfluid velocity is shown in Fig. 2(a). Heating of the dark part of the particle results in a rush of superflow towards the hotter part and steady propulsion of the particle. A further increase in $Q$ above a critical value results in a dramatic transition (Fig. 3 and Supplemental Video 1 [37]). The particle accelerates spontaneously, reaches a critical velocity, and then abrupt dissipation events occur. Multiple quantum vortices detach from the particle [Fig. 3(b)], followed by the intense generation of second sound waves [Fig. 3(c)]. The distribution of the superfluid velocity during the vortex generation event is shown in Fig. 3(e). One clearly sees a circulation around the quantum vortices (seen as dark blue points). During the dissipation events, the particle slows down and even recoils back for a short time [Figs. 3(f) and 3(g)]. In the course of the dissipation event, the particle also changes the orientation. It happens due to a significant redistribution of the superfluid velocity and, consequently, the traction force on the particle surface during vortex nucleation. These events occur with the rate increasing with the heat flux value $Q$ [Fig. 3(f)]. The vortices generated by the particle are often absorbed back by the particle during the recoil events when it moves backward. For larger $Q$ values, some vortex pairs detach and dwell in the bulk until mutual annihilation. Also, due to an interaction between the particle with vortices and sound waves, the particle direction during the dissipation events changes abruptly, resulting in seemingly jagged trajectories [Fig. 3(g)]. A somewhat similar randomlike behavior was observed in a mechanical system: a self-propelled oscillating droplet interacting with cylindrical surface waves [38]. 

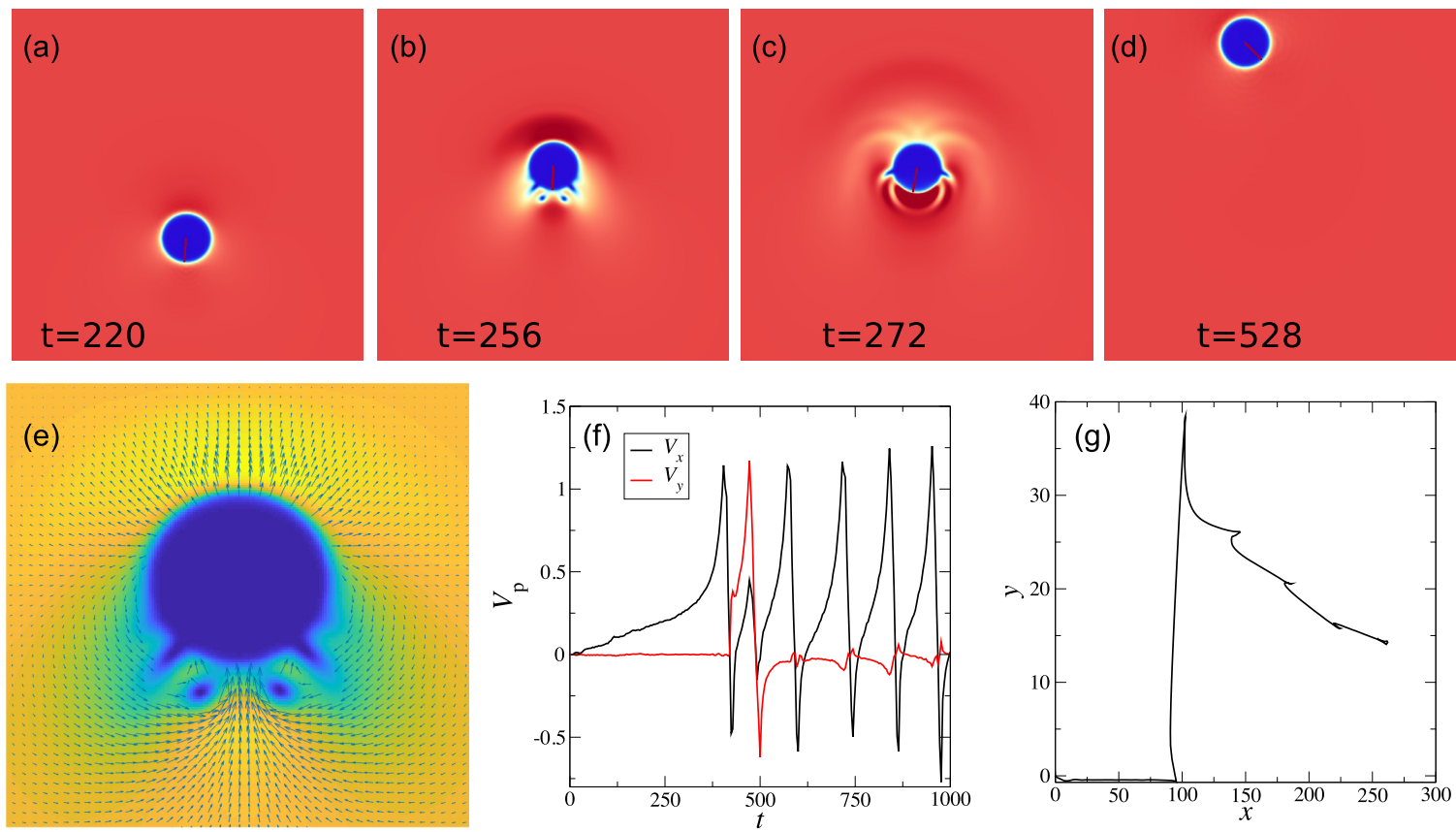

FIG. 3. (a)-(d) A sequence of snapshots illustrating unsteady particle motion for larger values of heat flux $Q$. The color code indicates the magnitude of the order parameter $|\psi|$. Red corresponds to $|\psi|=1$, and blue corresponds to $|\psi|=0$. The red stripe depicts the particle orientation, and the particle is shown as a blue circle. Only a small part of the entire integration domain is shown. The particle accelerates to the critical superfluid velocity until time $t=60$, generates vortices (seen as blue dots), second sound waves at $t=70$ (seen as bright stripes), and then slows down at $t=120$. (e) Blowup of (c) around the particle. Red arrows indicate the superfluid velocity. The color code is the same as in Fig. 2(a). (f) The particle velocity $V_{\mathrm{p}}$ vs time. (g) The particle trajectory. See also Supplemental Video 1 . Parameters: $\Lambda=0.1, Q=0.124$, $a=10 \xi, \tilde{\eta}=0.2$.

\section{B. Interaction between two particles}

The interaction between two superfluid swimmers is seemingly quite complex (see Fig. 4 and Supplemental Videos 2 and 3 [37]). Initially, two particles launched on parallel tracks attract each other. The initial attraction is a manifestation of Bernoulli's principle, similar to the attraction between two ships sailing on parallel tracks. In the course of an interaction, the particles accelerate and generate vortices and second sound waves. During this relatively short event, the particles turn away and speed out. Thus, the resulting interaction is repulsive.

\section{Simplified model for relaxation oscillations}

The numerical solution of the GLKE (4) and Newton equations for the particle Eqs. (6) and (7) exhibit stick-slip-like dynamics [39] [see Fig. 3(f)]. On the phenomenological level, this dynamics can be modeled in the framework of two coupled ordinary differential equations for the particle velocity $\mathbf{V}_{\mathrm{p}}$ and the integrated over the particle surface superfluid velocity $\mathbf{V}_{\mathrm{s}}$. Consider for simplicity a particle moving along the $x$ direction. The corresponding equations can be cast in the following form,

$$
\begin{aligned}
m_{\mathrm{p}} \frac{d V_{\mathrm{p}}}{d t} & =-\eta V_{\mathrm{p}}+F\left(V_{\mathrm{s}}\right), \\
\frac{d V_{\mathrm{s}}}{d t} & =R\left(V_{\mathrm{p}}, V_{\mathrm{s}}\right) .
\end{aligned}
$$

Here, the force $F \propto Q$ depends on the averaged superfluid velocity $V_{\mathrm{s}}$, and $R\left(V_{\mathrm{p}}, V_{\mathrm{s}}\right)$ describes the superfluid velocity evolution due to heating and particle displacement. We assume a powerlike dependence of $R, F$ on $V_{\mathrm{p}}, V_{\mathrm{s}}$ and keep only the leading terms:

$$
\begin{aligned}
& R=a_{0}+a_{1} V_{\mathrm{s}}+a_{2} V_{\mathrm{s}}^{2}+a_{3} V_{\mathrm{s}}^{3}+a_{4} V_{\mathrm{p}}, \\
& F=b_{1} V_{\mathrm{s}}+b_{2} V_{\mathrm{s}}^{2}+b_{3} V_{\mathrm{s}}^{3} .
\end{aligned}
$$

Here, the coefficients $a_{0}, a_{1}, a_{2}, a_{3}$ also depend on the heat flux. The term $a_{4} V_{\mathrm{p}}$ describes the expulsion of the superfluid by a moving solid object. Terms up to $V_{\mathrm{s}}^{3}$ are needed to suppress the unlimited acceleration of the superfluid and for saturation of the oscillations.

Equations (9) and (10) exhibit relaxation periodic oscillations in a broad range of parameters [see Fig. 4(e)]. The period of oscillations depends on the heat flux $Q$ : The period diverges for $Q \rightarrow 0$. Overall, the behavior of the reduced model on a qualitative level is reminiscent of full Eqs. (4), (6), and (7): periodic oscillations with a slow initial rise of the velocity and an abrupt drop after the velocity reaches a critical value. The reduced system even captures a recoil effect: After the acceleration event, the particle velocity $V_{\mathrm{p}}$ becomes negative for a short time period.

\section{Numerical implementation}

We solved the GLKE (4) by the quasispectral method based on the fast Fourier transformation $[35,40]$ in two di- 


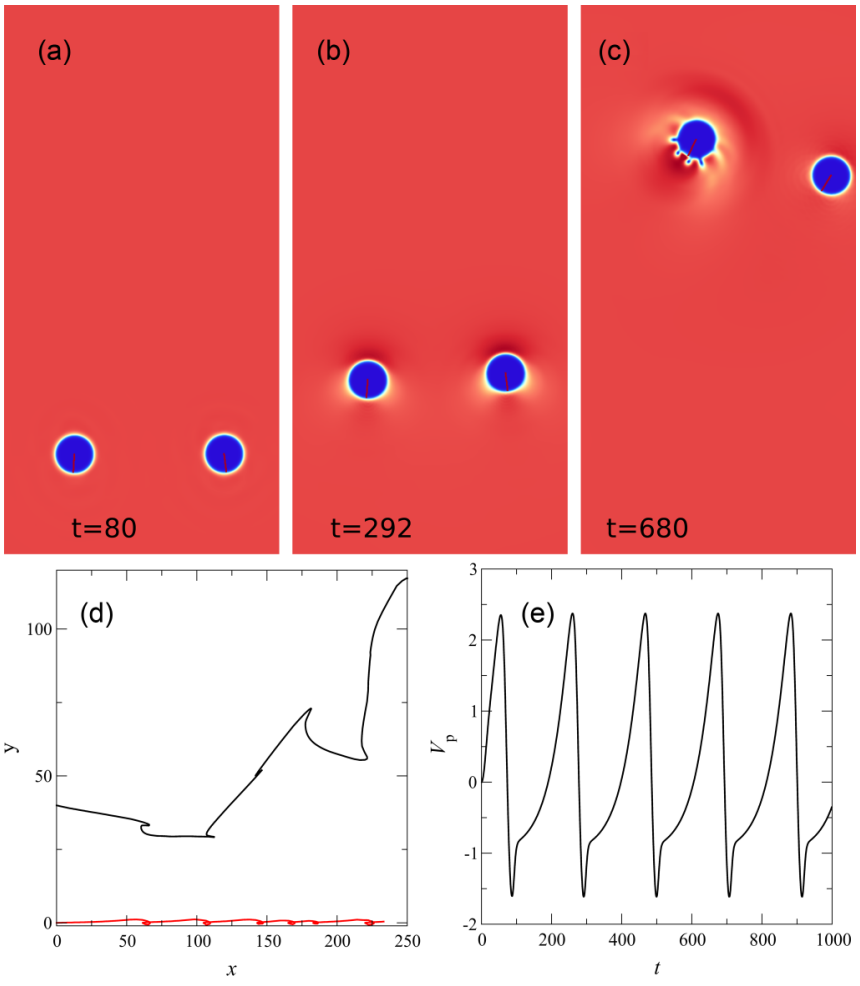

FIG. 4. Interactions of two particles. (a)-(c) A sequence of snapshots illustrating the interactions of two particles. The color code indicates the magnitude of the order parameter $|\psi|$. Red corresponds to $|\psi|=1$, and blue corresponds to $|\psi|=0$. The red stripe depicts the particle orientation. The initial attraction between the particles $(0<t<700)$ changed by repulsion for $t>770$ and the generation of vortices and the second sound. (d) The trajectories of the particles (particle 1 is black, particle 2 is red). Particles released at $x=0$ with parallel velocities and an initial separation distance of 80 numerical units. See also Supplemental Videos 2 and 3. The parameters are $\Lambda=$ $0.1, Q=0.122$. (e) Particle velocity $V_{\mathrm{p}}$ vs time in the reduced model (9) and (10) for $m_{\mathrm{p}}=10, \eta=0.01, F=4 V_{\mathrm{s}}\left(1-1.27 V_{\mathrm{s}}-0.84 V_{\mathrm{s}}^{2}\right)$, $R=0.015+0.08 V_{\mathrm{s}}\left(1-5 V_{\mathrm{s}}^{2}\right)-0.02 V_{\mathrm{p}}$.

mensions. The algorithm was implemented in the graphical processing unit (GPU). Typically, $2048 \times 2048$ mesh points were used in the periodic domain 600 coherence length $\xi$, and the particle radius was $10-20 \xi$. The time interval was over 2000 dimensionless units. The position of the particle was updated according to Eq. (6). The particle was implemented in Eq. (4) by the following method. The coefficient $b$ in front of the linear term $\psi$ in Eq. (4) was turned negative at the location of the particle (compare Ref. [41]). It forced the order parameter $\psi$ to rapidly decay to zero at the entire area occupied by the particle. Furthermore, it guaranteed that the perpendicular component of the superfluid velocity is zero on the particle surface. The traction force $\mathbf{P}_{\tau}$ over the dark face of the particle was implemented by the immersed boundary method [42]. The corresponding value of the superfluid velocity in the expression was obtained from the solution of Eq. (4).

\section{E. Parameter estimates}

For the heat flux $q \approx 0.1 \mathrm{~W} / \mathrm{cm}^{2}$ emitted from a solid, superfluid helium He-II is still laminar [43]. It is worth noting that the heat fluxes from the solid surface to He-II can reach $30 \mathrm{~W} / \mathrm{cm}^{2}$ before the superfluid boils at temperatures $T \sim 1.8-2.1 \mathrm{~K}$ [44]. To estimate the characteristic particle velocities, we take the temperature of experiment $T=2.1 \mathrm{~K}$, that is, $\approx 7 \times 10^{-2} \mathrm{~K}$ below the normal-to-superfluid transition temperature $T_{\lambda}$ at the saturated vapor pressure. Using $\rho=0.146 \mathrm{~g} / \mathrm{cm}^{3}$ and $\rho_{s}=3.78 \times 10^{-2} \mathrm{~g} / \mathrm{cm}^{3}$ for the total and superfluid densities, respectively, $S=0.184 \mathrm{~J} / \mathrm{cm}^{3} \mathrm{~K}$ for the entropy per unit of volume, and $\eta=1.80 \times 10^{-6} \mathrm{~Pa} \mathrm{~s}$ for the dynamic viscosity [45], one has the characteristic normal and superfluid velocities near the particle surface $v_{n} \sim q / S T \approx 2.3 \mathrm{~cm} / \mathrm{s}$ and $v_{s} \sim \rho_{n} v_{n} / \rho_{s} \approx 7.4 \mathrm{~cm} / \mathrm{s}$, and the tangential stress acting on the particle $P_{\tau} \sim \rho_{n} q v_{s} / S T \approx$ $0.21 \mathrm{~Pa}$. The particle velocity resulting from the balance of the traction and viscous drag from the normal component is estimated as $V_{\mathrm{p}} \sim P_{\tau} a / 6 \eta \sim 19 \mathrm{~cm} / \mathrm{s}$. In this estimate we assume that the heat flux density from the particle's surface is $q=1 \mathrm{~W} / \mathrm{cm}^{2}$ and the particle radius is $a=10 \mu \mathrm{m}$.

For the temperatures $T_{\lambda}-T \approx 10^{-3}$, we obtain a coherence length in the range $\xi \approx 20-40 \mathrm{~nm}$, and a particle radius of the order 200-400 nm. Correspondingly, the domain size is 10-20 $\mu \mathrm{m}$. Thus, the computational particle size is smaller than a typical size of a tracer $1-10 \mu \mathrm{m}$. Performing calculations with realistic particle sizes is not feasible due to hardware limitations. However, it is unlikely that qualitatively different phenomena can arise with the increase of the particle and domain sizes.

\section{CONCLUSION}

In our consideration, we neglected particle sedimentation due to the superfluid/particle density difference. Since for typical particle sizes of the order of $1 \mu \mathrm{m}$, the self-propulsion velocity exceeds the sedimentation velocity $(\sim 0.1 \mathrm{~cm} / \mathrm{s})$, the effect of gravity can be neglected.

We considered the self-propelled motion of colloidal microparticles in superfluid helium-II. For the sake of simplicity, we studied the dynamics in the vicinity of the $\lambda$ point where the superfluid can be effectively described by the GLKE. Our studies revealed complex particle-fluid interactions: spontaneous accelerations terminated by short dissipation events when vortices and the second sound are generated. We also observed that the swimmers' interaction is more subtle than that between passive inclusions: An initial attraction precedes vigorous repulsion. The mechanism of self-propulsion discussed here is generic and applicable even far from the $\lambda$ point. The results apply to other superfluidlike systems, such as the $B$ phase of superfluid He-I [40], and Bose-Einstein and similar ultracold gas condensates.

An extremely low density of liquid helium (about $0.125 \mathrm{~g} / \mathrm{cm}^{3}$ ) imposes some limitations on the choice of swimming particles. Most solid materials will sediment. However, hollow glass spheres are promising candidates [46]. They were used as neutrally buoyant tracers in the experiments on liquid-helium turbulence [47]. Helium penetration 
of the microporous hollow glass surface is over $10 \mathrm{~h}$ and significantly exceeds the time of the experiment [48]. Janus particles can be fabricated by the evaporation of the desired metals - nickel, platinum, or gold - onto the particles using a direct line-of-sight approach [5]. Finally, hydrogen/deuterium ice microcrystals can also be used as tracers [49].

\section{ACKNOWLEDGMENT}

The research of I.S.A. was supported by the U.S. Department of Energy, Office of Science, Basic Energy Sciences, under Award No. DE-SC0020964.
[1] E. M. Purcell, Life at low Reynolds number, Am. J. Phys. 45, 3 (1977).

[2] T. Y.-T. Wu, Hydromechanics of swimming propulsion. Part 1. Swimming of a two-dimensional flexible plate at variable forward speeds in an inviscid fluid, J. Fluid Mech. 46, 337 (1971).

[3] E. Kanso, Swimming in an inviscid fluid, Theor. Comput. Fluid Dyn. 24, 201 (2010).

[4] H. Saito, Can we swim in superfluids?: Numerical demonstration of self-propulsion in a Bose-Einstein condensate, J. Phys. Soc. Jpn. 84, 114001 (2015).

[5] R. D. Baker, T. Montenegro-Johnson, A. D. Sediako, M. J. Thomson, A. Sen, E. Lauga, and I. S. Aranson, Shapeprogrammed 3D printed swimming microtori for the transport of passive and active agents, Nat. Commun. 10, 4932 (2019).

[6] W. F. Paxton, A. Sen, and T. E. Mallouk, Motility of catalytic nanoparticles through self-generated forces, Chem. Eur. J. 11, 6462 (2005).

[7] A. A. Solovev, Y. Mei, E. Bermúdez Ureña, G. Huang, and O. G. Schmidt, Catalytic microtubular jet engines self-propelled by accumulated gas bubbles, Small 5, 1688 (2009).

[8] W. Wang, W. Duan, Z. Zhang, M. Sun, A. Sen, and T. E. Mallouk, A tale of two forces: Simultaneous chemical and acoustic propulsion of bimetallic micromotors, Chem. Commun. 51, 1020 (2015).

[9] S. Sengupta, K. K. Dey, H. S. Muddana, M. E. Tabouillot, T Ibele, P. J. Butler, and A. Sen, Enzyme molecules as nanomotors, J. Am. Chem. Soc 135, 1406 (2013).

[10] H.-R. Jiang, N. Yoshinaga, and M. Sano, Active Motion of a Janus Particle by Self-Thermophoresis in a Defocused Laser Beam, Phys. Rev. Lett. 105, 268302 (2010).

[11] S. J. Ebbens and J. R. Howse, In pursuit of propulsion at the nanoscale, Soft Matter 6, 726 (2010).

[12] J. Palacci, S. Sacanna, A. P. Steinberg, D. J. Pine, and P. M. Chaikin, Living crystals of light-activated colloidal surfers, Science 339, 936 (2013).

[13] R. Golestanian, Collective Behavior of Thermally Active Colloids, Phys. Rev. Lett. 108, 038303 (2012).

[14] I. M. Khalatnikov, An Introduction to the Theory of Superfluidity (W. A. Benjamin, New York, 1965).

[15] T. Frisch, Y. Pomeau, and S. Rica, Transition to Dissipation in a Model of Superflow, Phys. Rev. Lett. 69, 1644 (1992).

[16] C. Josserand, Y. Pomeau, and S. Rica, Cavitation Versus Vortex Nucleation in a Superfluid Model, Phys. Rev. Lett. 75, 3150 (1995).

[17] I. Aranson and V. Steinberg, Stability of multicharged vortices in a model of superflow, Phys. Rev. B 53, 75 (1996).

[18] I. Aranson and V. Steinberg, Spin-up and nucleation of vortices in superfluid ${ }^{4}$ He, Phys. Rev. B 54, 13072 (1996).

[19] S. Musser, D. Proment, M. Onorato, and W. T. M. Irvine, Starting Flow Past an Airfoil and its Acquired
Lift in a Superfluid, Phys. Rev. Lett. 123, 154502 (2019).

[20] A. N. Ganshin, V. B. Efimov, G. V. Kolmakov, L. P. MezhovDeglin, and P. V. E. McClintock, Observation of an Inverse Energy Cascade in Developed Acoustic Turbulence in Superfluid Helium, Phys. Rev. Lett. 101, 065303 (2008).

[21] D. P. Meichle and D. P. Lathrop, Nanoparticle dispersion in superfluid helium, Rev. Sci. Instrum. 85, 073705 (2014).

[22] L. P. Pitaevskii, Phenomenological theory of superfluidity near the $\lambda$ point, Sov. Phys.-JETP 35, 282 (1959).

[23] L. D. Landau and E. M. Lifshitz, Fluid Mechanics (Butterworth and Heinemann, Oxford, UK, 1987).

[24] G. Gompper, R. G. Winkler, T. Speck, A. Solon, C. Nardini, F. Peruani, H. Löwen, R. Golestanian, U. B. Kaupp, L. Alvarez et al., The 2020 motile active matter roadmap, J. Phys.: Condens. Matter 32, 193001 (2020).

[25] C. Bechinger, R. Di Leonardo, H. Löwen, Ch. Reichhardt, G. Volpe, and G. Volpe, Active particles in complex and crowded environments, Rev. Mod. Phys. 88, 045006 (2016).

[26] R. Piazza and A. Parola, Thermophoresis in colloidal suspensions, J. Phys.: Condens. Matter 20, 153102 (2008).

[27] J. Wilks, The Properties of Liquid and Solid Helium (Clarendon, Oxford, UK, 1967).

[28] L. D. Landau and E. M. Lifshitz, Fluid Mechanics (Pergamon, Oxford, UK, 1959).

[29] I. Yu. Borisenko, V. B. Efimov, and L. P. Mezhov-Deglin, Nonlinear second-sound waves in a liquid helium resonator, Fizika Nizkih Temperatur 14, 1123 (1988).

[30] A. Y. Iznankin and L. P. Mezhov-Deglin, Shock waves in liquid helium, Sov. Phys.-JETP 57, 801 (1983).

[31] V. B. Efimov, G. V. Kolmakov, E. V. Lebedeva, L. P. MezhovDeglin, and A. B. Trusov, Generation of second and first sound waves by a pulse heater in He-II under pressure, J. Low Temp. Phys. 119, 309 (2000).

[32] A. Phillips and P. V. E. McClintock, Field emission and field ionization in liquid ${ }^{4} \mathrm{He}$, Philos. Trans. R. Soc. A 278, 271 (1975).

[33] A. I. Golov, P. M. Walmsley, and P. A. Tompsett, Charged tangles of quantized vortices in superfluid ${ }^{4} \mathrm{He}$, J. Low Temp. Phys. 161, 509 (2010)

[34] G. G. Ihas and F. Pobell, Correlation length, finite-geometry effects, and universality in pressurized superfluid helium near $T_{\lambda}$, Phys. Rev. A 9, 1278 (1974).

[35] A. Glatz, H. L. L. Roberts, I. S. Aranson, and K. Levin, Nucleation of spontaneous vortices in trapped Fermi gases undergoing a BCS-BEC crossover, Phys. Rev. B 84, 180501(R) (2011).

[36] P. Scherpelz, K. Padavić, A. Rançon, A. Glatz, I. S. Aranson, and K. Levin, Phase Imprinting in Equilibrating Fermi Gases: The Transience of Vortex Rings and Other Defects, Phys. Rev. Lett. 113, 125301 (2014). 
[37] See Supplemental Material at http://link.aps.org/supplemental/ 10.1103/PhysRevResearch.3.013188 for the legends to the Supplemental Videos.

[38] M. Hubert, S. Perrard, M. Labousse, N. Vandewalle, and Y. Couder, Tunable bimodal explorations of space from memorydriven deterministic dynamics, Phys. Rev. E 100, 032201 (2019).

[39] D. Volfson, L. S. Tsimring, and I. S. Aranson, Stick-slip dynamics of a granular layer under shear, Phys. Rev. E 69, 031302 (2004).

[40] I. S. Aranson, N. B. Kopnin, and V. M. Vinokur, Nucleation of Vortices by Rapid Thermal Quench, Phys. Rev. Lett. 83, 2600 (1999).

[41] M. M. Genkin, A. Sokolov, and I. S. Aranson, Spontaneous topological charging of tactoids in a living nematic, New J. Phys. 20, 043027 (2018).

[42] C. S. Peskin, The immersed boundary method, Acta Numer. 11, 479 (2002).

[43] J. L. Olsen, Optical observations of second sound in liquid helium II, Physica 69, 136 (1973).

[44] V. B. Efimov, A. N. Ganshin, G. V. Kolmakov, P. V. E. McClintock, and L. P. Mezhov-Deglin, Acoustic turbulence in superfluid ${ }^{4} \mathrm{He}$, J. Low Temp. Phys. 156, 95 (2009).
[45] R. J. Donnelly and C. F. Barenghi, The observed properties of liquid helium at the saturated vapour pressure, J. Phys. Chem. Ref. Data 27, 1217 (1998).

[46] G. P. Bewley, K. R. Sreenivasan, and D. P. Lathrop, Particles for tracing turbulent liquid helium, Exp. Fluids 44, 887 (2008).

[47] R. J. Donnelly, A. N. Karpetis, J. J. Niemela, K. R. Sreenivasan, W. F. Vinen, and C. M. White, The use of particle image velocimetry in the study of turbulence in liquid helium, J. Low Temp. Phys. 126, 327 (2002).

[48] I. V. Kazanin, V. N. Zinoviev, V. A. Lebiga, A. Y. Pak, V. M. Fomin, A. S. Vereshchagin, and N. G. Tsibulsky, Experimental investigation of transport and helium permeability for hollow microspherical membranes, in Proceedings of the XXV Conference on High-Energy Processes in Condensed Matter (HEPCM 2017): Dedicated to the 60th anniversary of the Khristianovich Institute of Theoretical and Applied Mechanics SB RAS, edited by V. Fomin, AIP Conf. Proc. No. 1893 (AIP, New York, 2017), p. 030013.

[49] W. Guo, M. La Mantia, D. P. Lathrop, and S. W. Van Sciver, Visualization of two-fluid flows of superfluid helium-4, Proc. Natl. Acad. Sci. USA 111, 4653 (2014). 